\title{
Solving 1D Conservation Laws Using Pontryagin's Minimum Principle
}

\author{
Wei Kang • Lucas C. Wilcox
}

October 16, 2018

\begin{abstract}
This paper discusses a connection between scalar convex conservation laws and Pontryagin's minimum principle. For flux functions for which an associated optimal control problem can be found, a minimum value solution of the conservation law is proposed. For scalar space-independent convex conservation laws such a control problem exists and the minimum value solution of the conservation law is equivalent to the entropy solution. This can be seen as a generalization of the Lax-Oleinik formula to convex (not necessarily uniformly convex) flux functions. Using Pontryagin's minimum principle, an algorithm for finding the minimum value solution pointwise of scalar convex conservation laws is given. Numerical examples of approximating the solution of both space-dependent and spaceindependent conservation laws are provided to demonstrate the accuracy and applicability of the proposed algorithm. Furthermore, a MATLAB routine using Chebfun is provided (along with demonstration code on how to use it) to approximately solve scalar convex conservation laws with space-independent flux functions.
\end{abstract}

Keywords Conservation laws · Pontryagin's minimum principle · Spectral method · Burgers' equation

\section{Introduction}

Conservation laws arise naturally from continuum physics. Scalar convex conservation laws have been studied extensively, developing both theory and numerical methods, see for example Dafermos [7], Evans [10], Lax [14], LeVeque [15], and the references there in. Many of the approaches taken, both theoretical and numerical, use the conservation law form of the problem. However, for some applications, such as landform evolution [18] and traffic flow [19], it can be natural to consider the integrated unknown from the conservation law. This leads to a Hamilton-Jacobi equation. This connection between conservation laws and Hamilton-Jacobi equations has been exploited in developing numerical algorithms for

This document has been approved for public release; its distribution is unlimited.

Wei Kang · Lucas C. Wilcox

Department of Applied Mathematics, Naval Postgraduate School, Monterey, CA

E-mail: \{wkang,lwilcox $\} @ n p s . e d u$ 
Hamilton-Jacobi equations [21, 22], see Osher and Fedkiw [20. Chapter 5] for a description of this history. The Hamilton-Jacobi equation can be solved using the method of characteristics. When characteristics cross a minimization principle is used by Luke [18] and Newell [19] to select a unique physically meaningful solution. Daganzo [8] has shown that this minimization procedure provides a stable solution. Sharing the similar idea of Hamilton-Jacobi equation and minimization, in this work we take a different approach in the study of a general family of conservation laws and initial conditions. Motivated by the the causality free method of solving Hamilton-Jacobi-Bellman equations by Kang and Wilcox [12, 13], we explore the connections between the entropy solution of scalar convex conservation laws and optimal control theory as well as the associated Pontryagin's minimum principle, which leads to an efficient numerical method for solving the conservation law.

Corrias et al. [6] proved that the entropy solution of a scalar convex conservation law is the gradient of the viscosity solution of an associated Hamilton-Jacobi equation. This underlying relationship was also used to find the numerical solution of conservation laws with large time steps [24]. This paper extends this connection further and introduces optimal control problems related to conservation laws. When an associated optimal control problem can be found, which is always the case for conservation laws with space-independent flux functions, Pontryagin's minimum principle gives a set of necessary conditions which can be used, along with the cost function, in an algorithm to find a minimum value solution of the conservation law. For conservation laws with space-independent flux functions, we show that this minimum value solution is indeed the entropy solution. This generalizes the Lax-Oleinik formula (see for example Evans [10, Section 3.4.2]) to convex (not necessarily uniformly convex) flux functions.

An alternative to the method proposed in this paper is the recent work of Darbon and Osher [9] and Chow et al. [4], which solves the Hopf formula to find the viscosity solution of Hamilton-Jacobi-Bellman equations. The algorithm is causality free and it is effective in solving high dimensional problems. The algorithm given by Darbon and Osher [9], like the one explored in this paper, also has an interesting link to conservation laws. For problems with a space-independent Hamiltonian and a convex initial condition, the algorithm converges to not only the solution of the Hamilton-Jacobi-Bellman equations, but also its gradient, which is a entropy solution of the corresponding conservation law.

The necessary conditions from Pontryagin's minimum principle are a set of boundary value problems that can be solved numerically using various techniques, we use a spectral collocation method provided by the Chebfun software package [1]. Although the solution of a conservation law is the gradient of the value function of an optimal control, Pontryagin's minimum principle contains this gradient as a part of its solution so that numerical differentiation is not necessary. For space-independent flux functions these necessary conditions become algebraic equations, which can be approximately solved using piecewise polynomial functions; again we use Chebfun in our implementation of the algorithm. For this case, we provide code for a numerical implementation of solving scalar convex conservation laws with space-independent flux functions pointwise. The algorithm does not need a grid in space and time. It achieves high accuracy even around shocks. In both space-dependent and -independent cases, minimizing the cost function provides a unique solution of the control problem and its gradient provides a pointwise solution of the conservation law. We finish our discussion with numerical examples demonstrating accuracy and applicability of the proposed algorithm where scalar convex conservation laws, such as Burgers' equation, are solved and compared to analytical or numerical solutions using other techniques. 


\section{From Pontryagin's minimum principle to conservation law}

In this section, we outline the underlying relationship between Pontryagin's minimum principle (PMP) and the solution of a conservation law. Consider the conservation law

$$
\begin{array}{rlrl}
u_{t}+(F(x, u))_{x}=0 & & \text { in } \mathbb{R} \times(0, T) \\
u & =g & & \text { on } \mathbb{R} \times\{t=0\},
\end{array}
$$

where the flux function $F: \mathbb{R} \times \mathbb{R} \rightarrow \mathbb{R}$ and initial condition $g: \mathbb{R} \rightarrow \mathbb{R}$ are given and $u: \mathbb{R} \times(0, T) \rightarrow \mathbb{R}$ is the unknown, $u=u(x, t)$. The associated Hamilton-Jacobi (HJ) equation has the following form

$$
\begin{aligned}
w_{t}+F\left(x, w_{x}\right) & =0 & & \text { in } \mathbb{R} \times(0, T) \\
w & =G & & \text { on } \mathbb{R} \times\{t=0\},
\end{aligned}
$$

where the initial condition $G: \mathbb{R} \rightarrow \mathbb{R}$ is such that

$$
G^{\prime}=g \quad \text { almost everywhere in } \mathbb{R}
$$

and $w: \mathbb{R} \times(0, T) \rightarrow \mathbb{R}$ is the unknown, $w=w(x, t)$. At any point where $w(x, t)$ has second order derivatives, we have

$$
u(x, t)=w_{x}(x, t)
$$

is a solution of the conservation law (1). Given the initial condition, $(x, s) \in \mathbb{R} \times[0, T]$, consider a related problem of optimal control

$$
\min _{\boldsymbol{\alpha}} J_{x, s}[\boldsymbol{\alpha}]=\min _{\boldsymbol{\alpha}} \int_{s}^{T} L(\boldsymbol{x}(r), \boldsymbol{\alpha}(r)) d r+G(\boldsymbol{x}(T)),
$$

where the response of the system, $\boldsymbol{x}:[s, T] \rightarrow \mathbb{R}$, is subject to

$$
\dot{\boldsymbol{x}}(r)=\boldsymbol{\alpha}(r), \quad \boldsymbol{x}(s)=x .
$$

$\boldsymbol{\alpha}:[0, T] \rightarrow A$ is a measurable function that represents the control input; $A$ is a subset of $\mathbb{R} ;$ $J_{x, s}: A \rightarrow \mathbb{R}$ is the cost function; and $L: \mathbb{R} \times A \rightarrow \mathbb{R}$ is called the Lagrangian. Define the Hamiltonian, $H: \mathbb{R} \times \mathbb{R} \times A \rightarrow \mathbb{R}$ as

$$
H(x, p, \alpha)=p \alpha+L(x, \alpha)
$$

and the value function, $V: \mathbb{R} \times[0, T] \rightarrow \mathbb{R}$, as

$$
V(x, s)=\inf _{\boldsymbol{\alpha}} J_{x, s}[\boldsymbol{\alpha}] .
$$

Then, the associated Hamilton-Jacobi-Bellman (HJB) equation is

$$
\begin{aligned}
V_{s}+\min _{\alpha} H\left(x, V_{x}, \alpha\right)=0 & \text { in } \mathbb{R} \times(0, T) \\
V=G & \text { on } \mathbb{R} \times\{s=T\} .
\end{aligned}
$$

If we define $t=T-s$ and $w(x, t)=V(x, T-t)$ we can rewrite this HJB equation as

$$
\begin{aligned}
w_{t}(x, t)-\min _{\alpha} H\left(x, w_{x}, \alpha\right) & =0 & & \text { in } \mathbb{R} \times(0, T) \\
w & =G & & \text { on } \mathbb{R} \times\{t=0\} .
\end{aligned}
$$


Suppose the flux function and Hamiltonian are related such that

$$
F(x, p)=-\min _{\alpha} H(x, p, \alpha)
$$

then the HJB equation (7) is equivalent to the HJ equation (2). Therefore, solving the optimal control problem (4) leads us to a solution of the HJ equation (2); and then to a solution of the conservation law (1) through (3). We summarize this relation in the following proposition.

Proposition 1 Let $V(x, s)$ be the value function defined in (5) by the optimal control (4). Suppose the flux function, $F$, in the conservation law (1) and HJ equation (2) satisfies (8) and suppose $G^{\prime}(x)=g(x)$ almost everywhere. Then the function $w(x, t)=V(x, T-t)$ satisfies the HJ equation (2) and the function $u(x, t)=w_{x}(x, t)$ satisfies the conservation law (1) at all points where the second order derivatives of $w(x, t)$ exist.

A conservation law may have multiple solutions with non-smooth properties such as shock and rarefaction waves. Uniqueness has been proved in the literature for entropy solutions. The relationship between $w(x, t)$ in Proposition 1 and the entropy solution is addressed in Section 3 for a family of conservation laws with a convex flux functions. In general, we call $u(x, t)=w_{x}(x, t)$ the minimum value solution of the conservation law (1) with respect to the Lagrangian $L(x, \alpha)$. Please note that the minimum value solution is unique for a given Lagrangian at all points where $w(x, t)$ admits the second order derivatives. This uniqueness is due to the fact that the value function of a problem of optimal control is unique.

The problem of finding the minimum value solution for a conservation law boils down to solving the optimal control problem (4). Let $\boldsymbol{\alpha}^{*}(x, p)$ be a solution of the following minimization

$$
\min _{\alpha} H(x, p, \alpha) .
$$

From PMP, an optimal trajectory of the control problem (4) satisfies the following necessary conditions

$$
\begin{aligned}
\dot{\boldsymbol{x}}(r) & =\boldsymbol{\alpha}^{*}(\boldsymbol{x}(r), \boldsymbol{p}(r)) \\
\dot{\boldsymbol{p}}(r) & =-\frac{\partial H}{\partial x}\left(\boldsymbol{x}(r), \boldsymbol{p}(r), \boldsymbol{\alpha}^{*}(\boldsymbol{x}(r), \boldsymbol{p}(r))\right) \\
\boldsymbol{x}(s) & =x \\
\boldsymbol{p}(T) & =G^{\prime}(\boldsymbol{x}(T))
\end{aligned}
$$

in which the costate, $\boldsymbol{p}:[s, T] \rightarrow \mathbb{R}$, corresponds the gradient of the optimal cost, i.e.,

$$
\boldsymbol{p}(r)=V_{x}(\boldsymbol{x}(r), r)
$$

Because all functions do not explicitly depend on $r$, we can shift $s$ to 0 so that the boundary value problem 10 is equivalent to

$$
\begin{aligned}
\dot{\boldsymbol{x}}(r) & =\boldsymbol{\alpha}^{*}(\boldsymbol{x}(r), \boldsymbol{p}(r)) \\
\dot{\boldsymbol{p}}(r) & =-\frac{\partial H}{\partial x}\left(\boldsymbol{x}(r), \boldsymbol{p}(r), \boldsymbol{\alpha}^{*}(\boldsymbol{x}(r), \boldsymbol{p}(r))\right) \\
\boldsymbol{x}(0) & =x \\
\boldsymbol{p}(t) & =G^{\prime}(\boldsymbol{x}(t))
\end{aligned}
$$


where $t=T-s$. If $G^{\prime}(x)$ does not exist at $a_{k}$, for $k=1,2, \ldots, m$, an optimal trajectory may satisfy the following equations

$$
\begin{aligned}
\dot{\boldsymbol{x}}(r) & =\boldsymbol{\alpha}^{*}(\boldsymbol{x}(r), \boldsymbol{p}(r)) \\
\dot{\boldsymbol{p}}(r) & =-\frac{\partial H}{\partial x}\left(\boldsymbol{x}(r), \boldsymbol{p}(r), \boldsymbol{\alpha}^{*}(\boldsymbol{x}(r), \boldsymbol{p}(r))\right) \\
\boldsymbol{x}(0) & =x \\
\boldsymbol{x}(t) & =a_{k}
\end{aligned}
$$

for $k=1,2, \ldots, m$. Both (11) and (12) are two-point boundary value problems (BVPs). If an optimal control problem can be derived in which the Lagrangian $L(x, \alpha)$ and its associated Hamiltonian that satisfies (8), then the following is a high-level algorithm for finding the minimum value solution based on the PMP. Note that this algorithm finds both the minimum value solution to the conservation law (1) and the solution to the associated HJ equation (2).

\section{Algorithm 1 (Minimum value solution)}

Step I For any given point $(x, t)$, find all solutions of the two-point BVPs (11) and [12].

Step II Among all the solutions in Step I, adopt the one, $(\boldsymbol{x}, \boldsymbol{p})$, with the smallest cost,

$$
J_{x, t}^{*}=\int_{0}^{t} L\left(\boldsymbol{x}(r), \boldsymbol{\alpha}^{*}(\boldsymbol{x}(r), \boldsymbol{p}(r))\right) d r+G(\boldsymbol{x}(t)) .
$$

Step III Set

$$
w(x, t)=V(x, T-t)=J_{x, t}^{*}, \quad u(x, t)=p(0) .
$$

For the rest of the paper, we address the following questions that are important to the algorithm.

- What is the relationship between the minimum value solution and the entropy solution?

- How to find a Lagrangian $L(x, \alpha)$ that satisfies 8 ?

- How to find all solutions of the two-point BVPs 11 and (12)?

In addition, several examples are shown in the following sections to test the algorithm for conservation laws with various types of flux functions.

\section{From viscosity solutions to entropy solutions}

This section describes the connection between the minimum value solution and the entropy for space-independent flux functions. The following assumptions are made in several theorems that follow.

Assumption 1 The initial condition function $g(x)$, in the conservation law (1), is bounded in $\mathbb{R}$ and it is continuous everywhere except for a finite number of points, $x=a_{1}, a_{2}, \ldots, a_{m}$.

Assumption 2 In the conservation law (1), the flux function $F(x, p)=F(p)$ is independent of $x$. In addition, $F$ is a convex function satisfying

$$
\lim _{|p| \rightarrow \infty} \frac{F(p)}{|p|}=+\infty
$$


Definition 1 Given a function $F: \mathbb{R} \rightarrow \mathbb{R}$, its Legendre transform is

$$
F^{*}(q)=\sup _{p}\{p q-F(p)\} \quad \text { for } q \in \mathbb{R} .
$$

For convex functions satisfying (13), the 'sup' in the definition can be replaced by 'max.' The following lemma formalizes when the Legendre transform of a convex function is a convex function and when the transform is an involution.

Lemma 1 (Convex duality [10]) Assume $F(p)$ satisfies Assumption 2] Then

(i) the mapping $q \rightarrow F^{*}(q)$ is convex and

$$
\lim _{|q| \rightarrow \infty} \frac{F^{*}(q)}{|q|}=+\infty
$$

(ii) Moreover

$$
F=\left(F^{*}\right)^{*}
$$

In this section, we consider the conservation law

$$
\begin{aligned}
u_{t}+(F(u))_{x} & =0 & & \text { in } \mathbb{R} \times(0, T) \\
u & =g & & \text { on } \mathbb{R} \times\{t=0\},
\end{aligned}
$$

in which $g$ and $F$ satisfy Assumptions 1 and 2 The associated HJ equation has the form

$$
\begin{aligned}
w_{t}+F\left(w_{x}\right) & =0 & & \text { in } \mathbb{R} \times(0, T) \\
w & =G & & \text { on } \mathbb{R} \times\{t=0\} \\
G^{\prime} & =g & & \text { almost everywhere in } \mathbb{R} .
\end{aligned}
$$

Following the idea in Section 2 the Hamiltonian of the optimal control problem must satisfy $(8)$. Let $F \circ(-1): \mathbb{R} \rightarrow \mathbb{R}$ represent the function

$$
p \rightarrow F(-p)
$$

Define the function $L: \mathbb{R} \rightarrow \mathbb{R}$ by

$$
L(\alpha)=(F \circ(-1))^{*}(\alpha)=\max _{p}\{p \alpha-F(-p)\} .
$$

Given the initial condition, $(x, s) \in \mathbb{R} \times[0, T]$, the associate problem of optimal control has the form

subject to

$$
\min _{\boldsymbol{\alpha}} J_{x, s}[\boldsymbol{\alpha}]=\min _{\boldsymbol{\alpha}} \int_{s}^{T} L(\boldsymbol{\alpha}(r)) d r+G(\boldsymbol{x}(T))
$$

$$
\dot{\boldsymbol{x}}(r)=\boldsymbol{\alpha}(r), \quad \boldsymbol{x}(s)=x .
$$

The Hamiltonian is independent of $x$, specifically

$$
H(p, \alpha)=p \alpha+L(\alpha) .
$$

The following lemma shows that the requirement $(8)$ is fulfilled.

Lemma 2 Suppose Assumption 2 holds. Then

$$
F(p)=-\min _{\alpha} H(p, \alpha) .
$$


Proof From Lemma1, we have

$$
F \circ(-1)=L^{*}
$$

More specifically, for any $p \in \mathbb{R}$

$$
\begin{aligned}
F(p) & =L^{*}(-p) \\
& =\max _{\alpha}\{(-p) \alpha-L(\alpha)\} \\
& =-\min _{\alpha}\{p \alpha+L(\alpha)\} \\
& =-\min _{\alpha} H(p, \alpha) .
\end{aligned}
$$

The following lemma will be used to simplify the form of the dynamics given in the PMP.

Lemma 3 Suppose $F \in C^{1}(\mathbb{R})$ satisfies Assumption 2 . Then the function

$$
\boldsymbol{\alpha}^{*}(p)=-F^{\prime}(p), \quad p \in \mathbb{R}
$$

is the unique solution of the following minimization

$$
\min _{\alpha} H(p, \alpha) .
$$

Proof From the definition (16),

$$
L(\alpha)=\max _{\lambda}\{\lambda \alpha-F(-\lambda)\}
$$

for all $\alpha \in \mathbb{R}$. Equivalently

$$
L(\alpha)=\lambda \alpha-F(-\lambda)
$$

where $\lambda$ is a number satisfying

$$
\alpha+F^{\prime}(-\lambda)=0 .
$$

For a given $\alpha$, the value of $\lambda$ satisfying (18) may not be unique. However, the minimum value, $L(\alpha)$, is unique because $\lambda \alpha-F(-\lambda)$ is convex. Define

$$
\begin{aligned}
\bar{H}(p, \lambda) & =H\left(p,-F^{\prime}(\lambda)\right) \\
& =-(p+\lambda) F^{\prime}(-\lambda)-F(-\lambda) .
\end{aligned}
$$

Assumption 2 implies that $F^{\prime}(-\lambda)$ is monotone and unbounded. Therefore, $\alpha$ minimizes $H(p, \alpha)$ if and only if there is a number $\lambda$ satisfying $[18$ that minimizes $\bar{H}(p, \lambda)$. To minimize $\bar{H}(p, \lambda)$, we consider

$$
\begin{aligned}
\bar{H}(p, \lambda) & =-(p+\lambda) F^{\prime}(-\lambda)-F(-\lambda)+F(p)-F(p) \\
& =-(p+\lambda) F^{\prime}(-\lambda)+(p+\lambda) F^{\prime}(\xi)-F(p) \\
& =-(p+\lambda)\left(F^{\prime}(-\lambda)-F^{\prime}(\xi)\right)-F(p)
\end{aligned}
$$

where $\xi$ is a number between $p$ and $-\lambda$. If $p+\lambda>0$, then $p>\xi>-\lambda$. We know that $F^{\prime}(\cdot)$ is nondecreasing. Therefore,

$$
-(p+\lambda)\left(F^{\prime}(-\lambda)-F^{\prime}(\xi)\right) \geq 0
$$


Similarly, we can prove the same inequality if $p+\lambda<0$. Therefore,

$$
\bar{H}(p, \lambda) \geq-F(p)=\bar{H}(p,-p),
$$

i.e., $\lambda=-p$ minimizes $\bar{H}(p, \lambda)$. Its minimum value is $-F(p)$. Therefore, $\alpha^{*}=-F^{\prime}(p)$ minimizes $H(p, \alpha)$.

To prove that $\alpha^{*}=-F^{\prime}(p)$ is the unique function that minimizes $H(p, \alpha)$, let us assume that $\alpha_{1}=-F^{\prime}\left(\lambda_{1}\right)$ minimizes $H(p, \alpha)$ and $\bar{H}(p, \lambda)$. Then

$$
\bar{H}\left(p, \lambda_{1}\right)=-F(p)
$$

i.e.,

$$
-\left(p+\lambda_{1}\right) F^{\prime}\left(-\lambda_{1}\right)-F\left(-\lambda_{1}\right)+F(p)=0 .
$$

Equivalently,

$$
F(p)=F\left(-\lambda_{1}\right)+\left(p+\lambda_{1}\right) F^{\prime}\left(-\lambda_{1}\right) .
$$

Because $F(\xi)$ is convex, its curve cannot lie below its tangent line. Therefore,

$$
F(\xi)=F\left(-\lambda_{1}\right)+\left(\xi+\lambda_{1}\right) F^{\prime}\left(-\lambda_{1}\right)
$$

for all $\xi$ between $\lambda_{1}$ and $p$. So,

$$
F^{\prime}(\xi)=F^{\prime}\left(-\lambda_{1}\right)
$$

Let $\xi=p$, then $-F^{\prime}(p)=-F^{\prime}\left(\lambda_{1}\right)=\alpha_{1}$. This implies that $\alpha^{*}=-F^{\prime}(p)$ is the unique function that minimizes $H(p, \alpha)$.

Using Lemma 3, the Hamilton dynamics (11a)- $11 \mathrm{~b}$ ) (and $(12 \mathrm{a}-(12 \mathrm{~b})$ ) for the control problem (17) is simplified to

$$
\begin{aligned}
& \dot{\boldsymbol{x}}(r)=\boldsymbol{\alpha}^{*}(p(r))=-F^{\prime}(p(r)) \\
& \dot{\boldsymbol{p}}(r)=0 .
\end{aligned}
$$

This implies that $\boldsymbol{p}$ is a constant and the characteristic is a straight line

$$
\begin{array}{ll}
\boldsymbol{p}(r) & \equiv p \quad \text { (a constant) } \\
\boldsymbol{x}(r) & =x-F^{\prime}(p) r .
\end{array}
$$

For this to be a solution of one of the two-point BVPs (11) and (12), $p$ must satisfy at least one of the following equations

$$
\begin{aligned}
p & =G^{\prime}\left(x-F^{\prime}(p) t\right) \\
a_{k} & =x-F^{\prime}(p) t \quad \text { for } 1 \leq k \leq m,
\end{aligned}
$$

where $a_{k}$ 's are the points at which $G^{\prime}(x)$, or $g(x)$, is discontinuous. Thanks to Assumption 2 , the two-point boundary value problem (BVP) of PMP boils down to the algebraic equations 20]. This is fundamentally different from the PMP in Section 2, where the problem is defined using differential equations. Under some conditions which will be addressed later, all solutions of the algebraic equations 20] can be found. 
Remark 1 From the Hamiltonian dynamics [19, the optimal trajectory is a line

$$
x+\alpha r
$$

where $\alpha=\boldsymbol{\alpha}(p)$ is a constant. The problem of optimal control [17) is equivalent to

$$
\min _{\alpha}\{L(\alpha) t+G(x+\alpha t)\}
$$

Define

$$
y=x+\alpha t
$$

then minimization problem (21), for $t>0$, is transformed to

$$
\min _{y}\left\{t L\left(\frac{y-x}{t}\right)+G(y)\right\}
$$

This is the Hopf-Lax formula [10].

Now, let us address the issue of discontinuity in solutions. Instead of a classic smooth solution, we consider the entropy solution of the conservation law (14). The problem is closely related to the viscosity solution of associated HJ equation. Suppose that the control input of the optimal control problem (17) is bounded and measurable, i.e., the set of possible control inputs is

$$
\left\{\boldsymbol{\alpha}:[0, T] \rightarrow\left[A_{1}, A_{2}\right] \mid \boldsymbol{\alpha} \text { is measurable }\right\}
$$

Theorem 1 ([10]) Suppose g satisfies Assumption 1 and $G$ is bounded. Further, suppose $F \in C^{1}(\mathbb{R})$ satisfies Assumption 2 Let $V(x, s)$ be the value function of the optimal control problem (17) with bounded control inputs and define

$$
w(x, t)=V(x, T-t) .
$$

Then, $w(x, t)$ is the viscosity solution of the initial value problem 15 .

It is proved by Corrias et al. [6] that the entropy solution of a conservation law is the gradient of the viscosity solution of the $\mathrm{HJ}$ equation.

Theorem 2 ([6]) Suppose $g$ satisfies Assumption 1$]$ Suppose $F \in C^{1}(\mathbb{R})$ satisfies Assumption 2. If $w \in W^{1, \infty}(\mathbb{R} \times(0, T])$ is the (unique) viscosity solution of the HJ equation [15], then $u=w_{x}$ is the (unique) entropy solution of the conservation law (14).

Theorems 1 and 2 almost bridge the HJ equation and the conservation law except that the control input is required to be bounded in 22]. In fact, it can be guaranteed that 22) holds true for the optimal control problem (17).

Proposition 2 Suppose that Assumptions 1 and 2 hold true. Then the value of optimal control, $\alpha^{*}$, for the optimal control problem (17) as a function of $x$ is bounded in $\mathbb{R}$.

Proof The result is trivially true if $F$ is $C^{1}$. However, it can be proved without this smoothness assumption. From the relation of the initial conditions (2c) and Assumption 1, we know that $G$ is Lipschitz. Let $C$ be its Lipschitz constant. Given $(x, s)$, the optimal control is a constant function, $\boldsymbol{\alpha}^{*} \equiv \alpha^{*}$ for some $\alpha^{*} \in \mathbb{R}$. The corresponding optimal cost value is

$$
J_{x, s}^{*}=\min _{\alpha}\{L(\alpha)(T-s)+G(x+(T-s) \alpha)\} .
$$


The optimal value is less than or equal to the value at $\alpha=1$, i.e.,

$$
L\left(\alpha^{*}\right)(T-s)+G\left(x+(T-s) \alpha^{*}\right) \leq L(1)(T-s)+G(x+(T-s)) .
$$

Because $G$ is Lipschitz

$$
\begin{aligned}
L\left(\alpha^{*}\right)(T-s)+G(x)-C(T-s)\left|\alpha^{*}\right| & \leq L\left(\alpha^{*}\right)(T-s)+G\left(x+(T-s) \alpha^{*}\right) \\
& \leq L(1)(T-s)+G(x+(T-s)) \\
& \leq L(1)(T-s)+G(x)+C(T-s) .
\end{aligned}
$$

Therefore,

$$
L\left(\alpha^{*}\right)-C\left|\alpha^{*}\right| \leq L(1)+C
$$

or equivalently

$$
\frac{L\left(\alpha^{*}\right)}{\left|\alpha^{*}\right|} \leq \frac{L(1)}{\left|\alpha^{*}\right|}+\frac{C}{\left|\alpha^{*}\right|}+C .
$$

Therefore, $\alpha^{*}$ must be bounded because of the following property of $L(\alpha)$

$$
\lim _{|\alpha| \rightarrow \infty} \frac{L(\alpha)}{|\alpha|}=+\infty \text {. }
$$

The following section contains a causality free algorithm for the computation of $u$ at any point $(x, t)$.

\section{A simplified numerical algorithm}

Let us consider a conservation law satisfying Assumptions 1 and 2 . It is proved in Section 3 that its entropy solution is the same as the minimum value solution with respect to an associated Lagrangian. In addition, the characteristics resulting from the PMP are straight lines. In this case, Algorithm 1 can be significantly simplified. In the following,

$$
L=(F \circ(-1))^{*} \text {. }
$$

If an explicit expression of this function is not derived, its value in 24 can be computed using the following formula

$$
L\left(-F^{\prime}(p)\right)=p F^{\prime}(p)-F(p) .
$$

because any $p$ satisfying $\alpha=-F^{\prime}(p)$ maximizes

$$
\max _{p}\{-p \alpha-F(p)\}
$$

which is equivalent to 16 .

Algorithm 2 (Entropy solution) This algorithm is for conservation laws satisfying Assumptions 1 and 2 . 
Step I Given any point $(x, t)$, find all values of $p$ that satisfy at least one of the algebraic equations 20 , i.e.,

$$
p=g\left(x-F^{\prime}(p) t\right)
$$

or

$$
a_{k}=x-F^{\prime}(p) t, \quad \text { for } 1 \leq k \leq m .
$$

Step II Among all the solutions in Step I, adopt the one with the smallest value,

$$
J^{*}=L\left(-F^{\prime}(p)\right) t+G\left(x-F^{\prime}(p) t\right)
$$

or equivalently

$$
J^{*}=\left(p F^{\prime}(p)-F(p)\right) t+G\left(x-F^{\prime}(p) t\right)
$$

Step III Set

$$
u(x, t)=p .
$$

Remark 2 Step I is critical in this algorithm. Given a general bounded initial function, $g$, the algebraic equations 23 may have multiple solutions. In the examples below we use piecewise-polynomial approximation [23] (and trigonometric approximation [29] for periodic functions) via Chebfun 1 [1] and its roots command to approximate the solutions of the algebraic equations 23. In the piecewise-polynomial case, the Chebfun command roots uses the algorithm described in Boyd [3]. Basically, it subdivides the interval into small pieces (based on the number of terms in the approximations) and uses the eigenvalues of the colleague matrix associated with the approximation on each interval [11, 26]. For further discussion of root finding using Chebfun see Trefethen [28 Chaper 18]. For the polynomial and trigonometric approximations used in this paper, we uses Chebfun's adaptive procedure to find the number of terms automatically to achieve roughly 15 digits of relative accuracy.

Here is an implementation of Algorithm 2] in MATLAB [27] using Chebfun.

\section{Implementation 1 (Entropy solution for convex flux functions)}

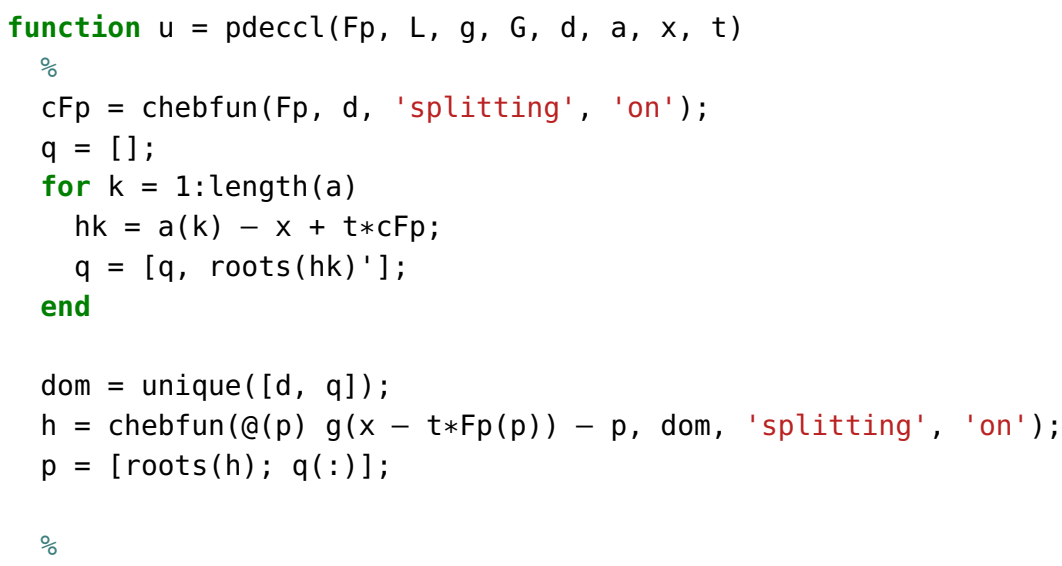

${ }^{1}$ Chebfun is a MATLAB [27] package that allows symbolic-like manipulation of functions at numerics speed using Chebyshev and Fourier series [1]. 


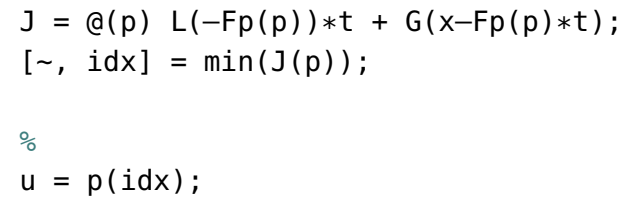

Here, $\mathrm{Fp}, \mathrm{L}, \mathrm{g}$, and $\mathrm{G}$ are the functions $F^{\prime}, L, g$, and $G$, respectively. The array $\mathrm{d}$ is the domain of definition (i.e., the range of $g$ including breakpoints where $F^{\prime}$ might not be smooth) that should be used with Chebfun and the array a contains the points at which the initial condition $g$ is discontinuous. The pair $(\mathrm{x}, \mathrm{t})$ is the point $(x, t)$ at which the solution $u$, returned as $\mathrm{u}$, of the conservation law is computed. If $g$ is a compactly supported chebfun ${ }^{2}$ (e.g., $g=$ chebfun $(\{0,1,0\},[-1,0,1,3]))$ and $F^{\prime}$ is smooth then $\mathrm{G}$, d, and a can be computed with:

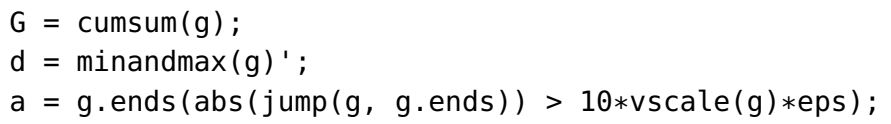

In this implementation, the Step I consists of lines 378 which solve the algebraic equations $23 \mathrm{~b}$ and lines 10,12 which find all the solutions of $23 \mathrm{a}$.

This algorithm has several advantages. In the presence of shock waves, finding the unique entropy solution is simple because the minimum value solution in Step II is unique. Furthermore, unlike the traditional method of characteristics for conservation laws, the computation does not explicitly use the Rankine-Hugoniot condition to calculate the behavior of the shock curves.

The algorithm is not based on interpolating and differentiation on a spacial grid and thus can avoid the Gibbs-Wilbraham phenomenon when calculating the solution at a point. We say that the algorithm is causality free or pointwise, meaning that the value of $u(x, t)$ is computed without using the value of $u$ at any other points. An advantage of causality free algorithms is their perfect parallelism. If the solution is to be computed at a large number of points, the computation is embarrassingly parallel.

Also because of the causality free property, the error does not propagate in space. The accuracy can be kept at the same level throughout a region and is based on the accuracy approximating the algebraic equations $(23)$ and finding their roots.

Remark 3 If we assume $t>0$ and that $F$ is uniformly convex, i.e., $F^{\prime \prime}(p)>\gamma>0$, then the algebraic equations [23) can be derived from the Lax-Oleinik formula in Evans [10] given here as

$$
u(x, t)=\left(F^{\prime}\right)^{-1}\left(\frac{x-\xi(x, t)}{t}\right)
$$

where

$$
\xi(x, t)=\underset{\xi}{\arg \min }\left\{t F^{*}\left(\frac{x-\xi}{t}\right)+G(\xi)\right\} .
$$

\footnotetext{
2 Chebfun with a capital $\mathrm{C}$ is the name of the software package, chebfun with a lowercase $\mathrm{c}$ is a single variable function defined on an interval created with the package [1].
} 
The critical points of the minimization problem in 25b must satisfy one of the following equations

$$
\left(F^{*}\right)^{\prime}\left(\frac{x-\xi}{t}\right)-g(\xi)=0
$$

or

$$
\xi=a_{k} \quad \text { for } 1 \leq k \leq m
$$

It can be proved that $\left(F^{*}\right)^{\prime}=\left(F^{\prime}\right)^{-1}$. Therefore, if we define

$$
\xi=x-F^{\prime}(p) t
$$

the minimizations (25b) and (24) are equivalent. Further, the equations for the critical points 26) are transformed to 23,

\section{Examples}

In the following, we illustrate Algorithms 1 and 2 using several examples with different types of flux functions, including both space-dependent and space-independent. A specific thing to note is the lack of Gibbs-Wilbraham oscillations in all of the approximate solutions given in the figures below, even in the presence of solutions with many shocks. All examples are computed using MATLAB 8.4.0.150421 (R2014b) and Chebfun 5.3.0 with double floatingpoint precision on an Apple MacBook Pro (Retina, 15-inch, Early 2013) with a $2.7 \mathrm{GHz}$ Intel Core i7 central processing unit and $16 \mathrm{~GB}$ of $1600 \mathrm{MHz}$ DDR3 random-access memory running OS X 10.10.5.

Remark 4 In the examples below we provide timings of Implementation 1 of the causalityfree Algorithm 2 Timings are also given for the second-order Lax-Wendroff finite volume method with the van Leer limiter from Clawpack ${ }^{3}[5,16]$ when it is compared with Implementation 1 These numbers show the current performance of our implementation. It should be noted that no performance tuning has been done by the authors for either code. In our experience if high-accuracy and/or the long time solution is desired at a few points in space and time then Implementation 1 is a competitive method. If lower-accuracy is okay and the solution is desired on a dense space-time grid then grid based methods, such as the finite-volume method, will be more competitive than Implementation 1

Example 1 We start by solving Burgers' equation

$$
\begin{aligned}
& u_{t}+\left(\frac{u^{2}}{2}\right)_{x}=0 \quad \text { in } \mathbb{R} \times(0, T) \\
& u=\left\{\begin{array}{ll}
1, & 0 \leq x \leq 1 \\
0, & \text { otherwise }
\end{array} \text { on } \mathbb{R} \times\{t=0\} .\right.
\end{aligned}
$$

\footnotetext{
3 More specifically, we used Clawpack v5.3.1-11-geb31727 from https://github.com/clawpack/ clawpack with the Chapter 11 examples in the git repository https://github.com/clawpack/apps (git commit ba557b49852377c05192d48289b3fbc8fea0f52e).
} 


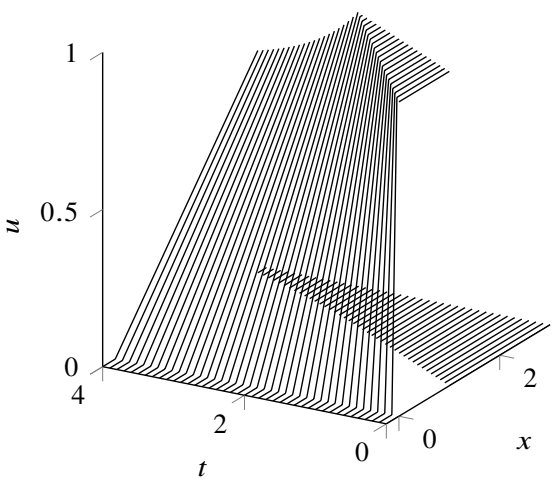

(a)

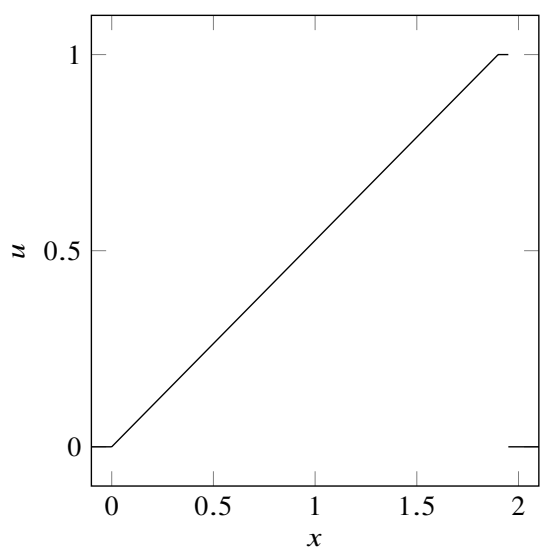

(b)

Fig. 1: The solution of Burgers' equation for the piecewise constant initial condition given in Example 1 Here (a) is a waterfall plot of the solution and $(\mathrm{b})$ is the solution for $t=1.9$.

It has a known solution given in Evans [10Example 3 of Chapter 3.4] as the following

$$
\begin{aligned}
& \text { for } 0 \leq t \leq 2 \\
& u(x, t)= \begin{cases}0, & x<0 \\
\frac{x}{t}, & 0<x<t \\
1, & t<x<1+\frac{t}{2} \\
0, & x>1+\frac{t}{2}\end{cases}
\end{aligned}
$$

and for $t \geq 2$

$$
u(x, t)= \begin{cases}0, & x<0 \\ \frac{x}{t}, & 0<x<(2 t)^{1 / 2} \\ 0, & x>(2 t)^{1 / 2} .\end{cases}
$$

The solution has shock wave which travel at two different speeds for $t<2$ and $t>2$, respectively. It also has a rarefaction wave for $t<2$. In the computation, we do not need any information about the shock wave speed.

The causality-free Algorithm 2 is applied using Implementation 1 . Note that for this simple case, a piecewise constant initial condition for Burgers' equation, the equations for the critical points $(23)$ can be solved directly and Chebfun is not required. However, we go ahead and use Chebfun anyways to benchmark the implementation. The maximum pointwise error (i.e., $l^{\infty}$ ) for the solution $u$ on a uniform grid of $100 \times 100$ points covering the domain $[-1,3] \times[0.1,4]$ (the point within $2.2204 \times 10^{-15}$ of the shock was excluded) is $2.2204 \times 10^{-16}$. For this error calculation, the solution is computed in parallel using a MATLAB parfor loop at the rate of 78 points per second (excluding the time for starting the parallel pool). Here $g$ and $\mathrm{G}$ are chebfuns, the code would be faster if these were simple MATLAB functions.

Implementation 1 provides a pointwise solution that we choose to feed back into Chebfun to build piecewise continuous approximations to the solution shown in Figure 1 The code used to generate this figure is given in Appendix A

Example 2 We solve Burgers' equation

$$
\begin{aligned}
u_{t}+\left(\frac{u^{2}}{2}\right)_{x} & =0 & & \text { in } \mathbb{R} \times(0, T) \\
u & =1+\sin (\pi x) & & \text { on } \mathbb{R} \times\{t=0\},
\end{aligned}
$$




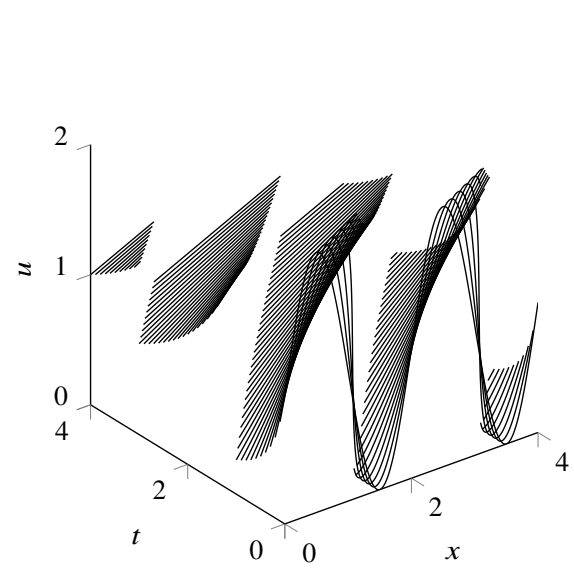

(a)

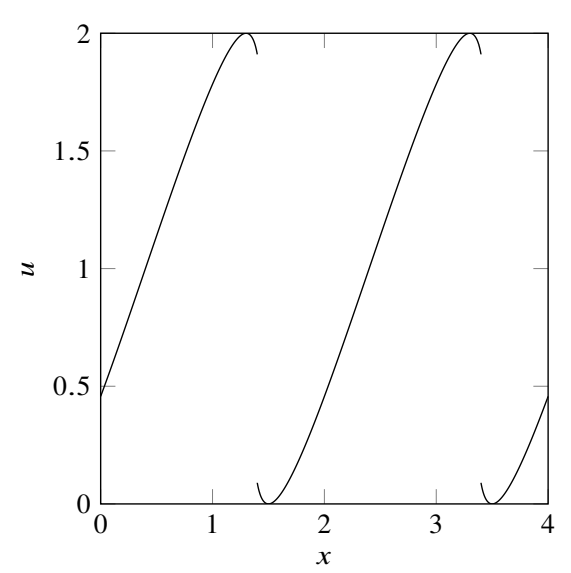

(b)

Fig. 2: The solution of Burgers' equation for the sinusoidal initial condition given in Example 2 Here (a) is a waterfall plot of the solution and (b) is the solution for $t=0.4$.

using Implementation 1 and compare the result against the traditional method of characteristics for conservation laws. The maximum pointwise error (i.e., $l^{\infty}$ ) for the solution $u$ on a uniform grid of $80 \times 80$ points covering the domain [0,4] $\times[0.1,0.8]$ is $1.2212 \times 10^{-14}$. For this error calculation, the solution is computed in parallel using a MATLAB parfor loop at the rate of 188 points per second (excluding the time for starting the parallel pool). Note, here we use MATLAB functions for $g$ and $\mathrm{G}$.

As in the previous example, we give the pointwise solution function to Chebfun and build piecewise continuous approximations to the solution shown in Figure 2

Example 3 We solve Burgers' equation with a compactly supported initial condition

$$
\begin{aligned}
u_{t}+\left(\frac{u^{2}}{2}\right)_{x} & =0 \\
u & =\left\{\begin{array}{ll}
(\cos (x)+1)\left(2 \sin (3 x)+\cos (2 x)+\frac{1}{5}\right), & -\pi \leq x \leq \pi \\
0, & \text { otherwise }
\end{array} \text { on } \mathbb{R} \times\{t=0\},\right.
\end{aligned}
$$

which is an example of $\mathrm{N}$-wave decay given in Chapter 11.5 of LeVeque [16]. As in the previous example, we give the pointwise solution function using Implementation 1 to Chebfun and build piecewise continuous approximations to the solution shown in Figure 3 . For verification, this figure also presents a solution to this problem using a second-order LaxWendroff finite volume method with the van Leer limiter from Clawpack 3 [5, 16] on a grid from $[-8,8]$ of 1000 cells, which took 0.021 seconds to compute to $t=1$.

Example 4 We solve Burgers' equation with a compactly supported initial condition with many oscillations

$$
\begin{aligned}
& u_{t}+\left(\frac{u^{2}}{2}\right)_{x}=0 \\
& \text { in } \mathbb{R} \times(0, T) \\
& u=\left\{\begin{array}{ll}
(\sin (x))^{2}+\sin \left(x^{2}\right), & 0 \leq x \leq 14 \\
0, & \text { otherwise }
\end{array} \text { on } \mathbb{R} \times\{t=0\},\right.
\end{aligned}
$$




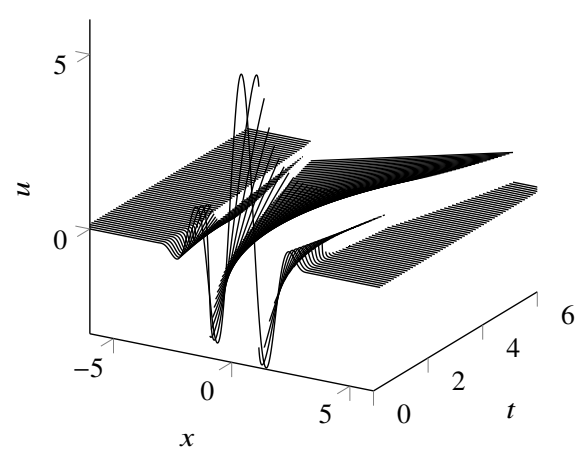

(a)

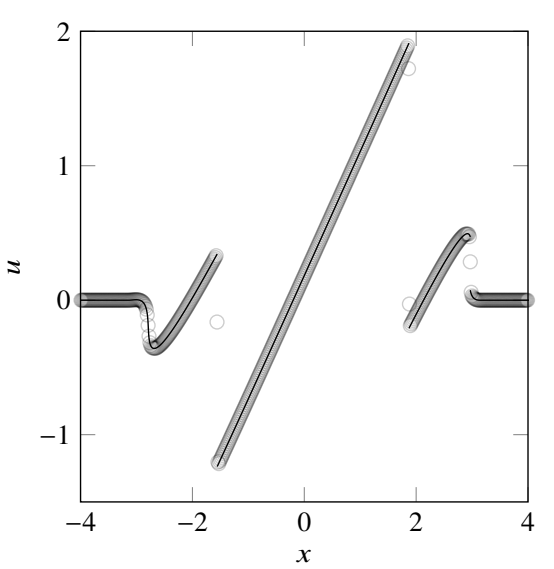

(b)

Fig. 3: The solution of Burgers' equation for the compactly supported initial condition given in Example 3. Here (a) is a waterfall plot of the solution and (b) is the solution for $t=1$ where the solid line is the chebfun constructed by the pointwise solution using Implementation 1 and the transparent circles show the computed solution using a finite volume method as described in Example 3

which we use to demonstrate the ability of the proposed algorithm to find solutions with many shocks. As in the previous example, we give the pointwise solution function using Implementation 1 to Chebfun and build piecewise continuous approximations to the solution shown in Figure 4 Figure 5 further illustrates the complexity of the solution.

Example 5 For this example consider a LWR model for traffic flow, named after Lighthill and Whitham [17], Richards [25] and Richards [25],

$$
\begin{aligned}
q_{t}+\left(v_{\max } q(1-q)\right)_{x} & =0 & & \text { in } \mathbb{R} \times(0, T), \\
q & =g & & \text { on } \mathbb{R} \times\{t=0\},
\end{aligned}
$$

where

$$
g(x)=\left\{\begin{array}{ll}
\frac{1}{5}+\frac{4}{5} \exp \left(-\frac{1}{20}\left(x-\frac{1}{3}\right)^{2}\right), & -30 \leq x \leq 30 \\
\frac{1}{5}, & \text { otherwise }
\end{array} \text { for } x \in \mathbb{R},\right.
$$

which is an example given in Chapter 11.1 of LeVeque [16]. Here $0 \leq q \leq 1$ is the density of the traffic flowing at a maximum speed $v_{\max }$. For this example we assume $v_{\max }=1$. The flux functions for this conservation law is concave upward and Algorithm 2 requires a conservation law with a convex downward flux function. Thus, we let $u=-q$ which transforms the LWR model 27] to the convex conservation law

$$
\begin{aligned}
u_{t}+\left(v_{\max } u(1+u)\right)_{x} & =0 & & \text { in } \mathbb{R} \times(0, T), \\
u & =-g & & \text { on } \mathbb{R} \times\{t=0\} .
\end{aligned}
$$

As in Example 3 we give the pointwise solution function using Implementation 1 to Chebfun and build piecewise continuous approximations to the solution shown in Figure 6 For 


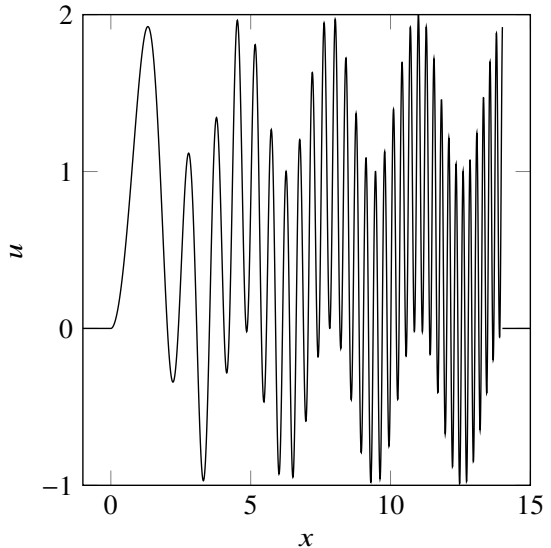

(a) $t=0$

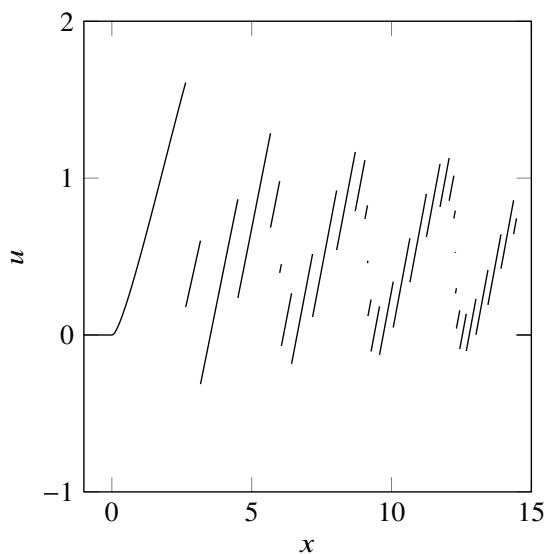

(c) $t=1$

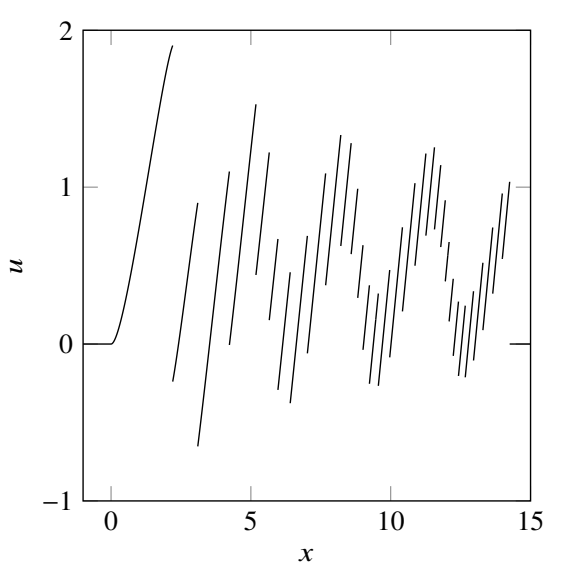

(b) $t=\frac{1}{2}$

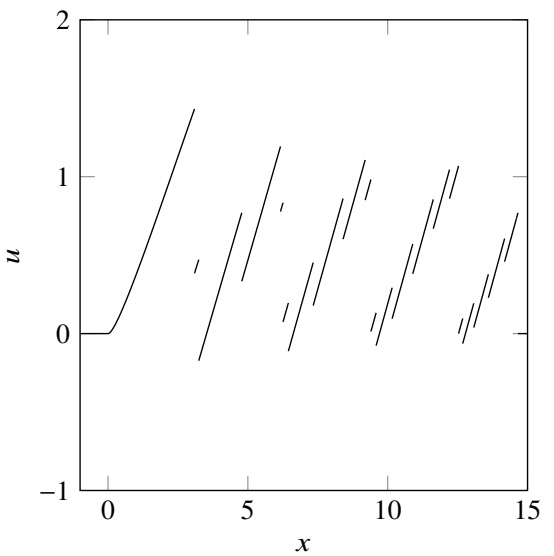

(d) $t=\frac{3}{2}$

Fig. 4: Various time instances of the solution of Burgers' equation for the compactly supported oscillatory initial condition given in Example 4

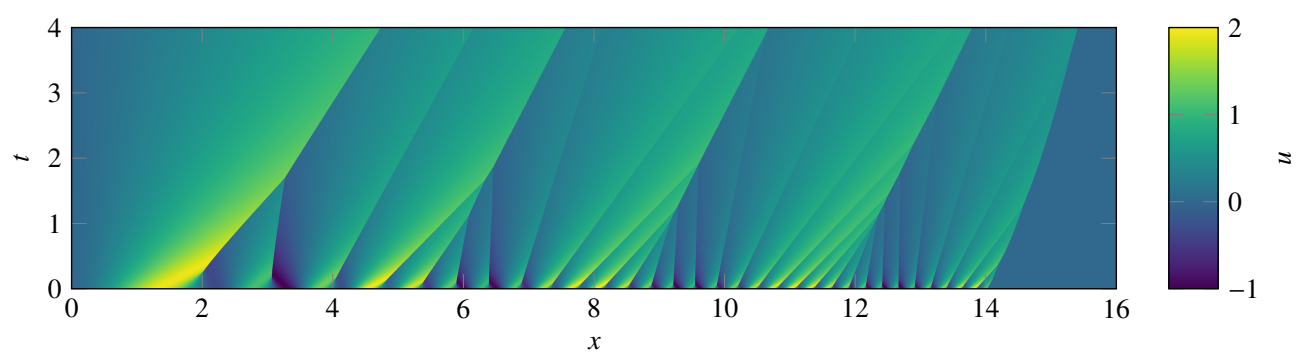

Fig. 5: The solution of Burgers' equation for the compactly supported oscillatory initial condition given in Example 4 


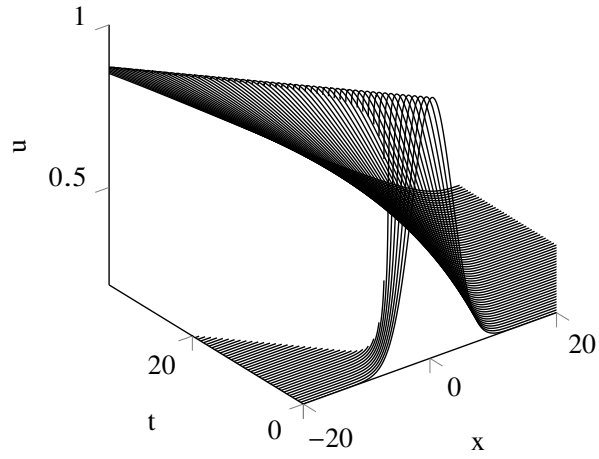

(a)

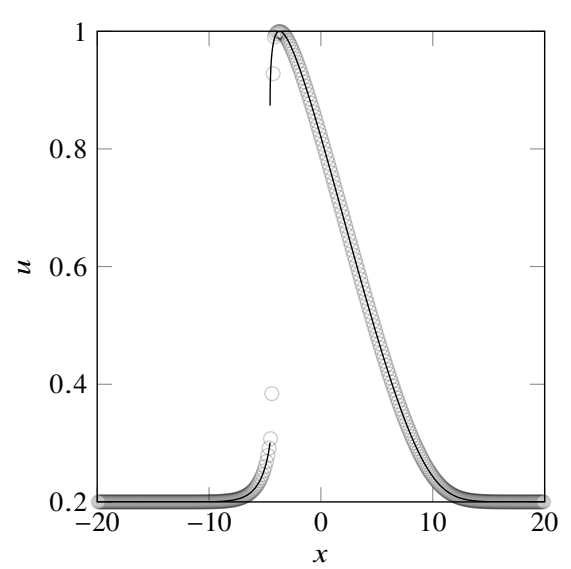

(b)

Fig. 6: The solution of the LWR equation for the initial condition given in Example 5 . Here (a) is a waterfall plot of the solution and (b) is the solution for $t=4$ where the solid line is the chebfun constructed by the pointwise solution using Implementation 1 and the transparent circles show the computed solution using a finite volume method as described in Example 5

verification, this figure also presents a solution to this problem using a second-order LaxWendroff finite volume method with the van Leer limiter from Clawpack $3[5,16]$ on a grid from $[-30,30]$ of 500 cells, which took 0.0024 seconds to reach $t=4$.

Example 6 In this example we consider the following conservation law with a spacedependent flux function

$$
\begin{aligned}
u_{t}+\left(\frac{u^{2}-x^{2}}{2}\right)_{x} & =0 & \text { in } \mathbb{R} \times(0, T), \\
u & = \begin{cases}1, & -1 \leq x \leq 0 \\
0, & \text { otherwise }\end{cases} & \text { on } \mathbb{R} \times\{t=0\} .
\end{aligned}
$$

Since the flux function is space-dependent, Algorithm 2 and the theory in Sections 3 and 4 do not apply. However, we can use Algorithm 1 to compute the unique minimum value solution. Some details required by the algorithm is given as follows. The associated Lagrangian is

$$
L(x, \alpha)=\frac{x^{2}+\alpha^{2}}{2}
$$

and the Hamiltonian is

$$
H(x, p, \alpha)=p \alpha+L(x, \alpha) .
$$

It is straightforward to derive relationship 8

$$
\frac{p^{2}-x^{2}}{2}=-\min _{\alpha} H(x, p, \alpha)
$$

and the associated optimal control 9

$$
\alpha^{*}=-p .
$$


Given any point $(x, t)$ we want to compute $u(x, t)$. The Hamilton dynamics in (11) and (12) have the following form

$$
\begin{aligned}
& \dot{\boldsymbol{x}}(r)=-\boldsymbol{p}(r), \\
& \dot{\boldsymbol{p}}(r)=-\boldsymbol{x}(r) .
\end{aligned}
$$

The solution with the initial condition $\boldsymbol{x}(0)=x$ is

$$
\begin{aligned}
& \boldsymbol{x}(r)=(x-C) e^{-r}+C e^{r}, \\
& \boldsymbol{p}(r)=(x-C) e^{-r}-C e^{r},
\end{aligned}
$$

where $C$ is an arbitrary constant. Denote $x(t)$ and $\boldsymbol{p}(t)$ by $X$ and $P$, respectively, then we have

$$
\begin{aligned}
& X=(x-C) e^{-t}+C e^{t}, \\
& P=(x-C) e^{-t}-C e^{t} .
\end{aligned}
$$

Solving for $C$ in terms of $X$ we have

$$
C=\frac{X-x e^{-t}}{e^{t}-e^{-t}}=2\left(X-x e^{-t}\right) \operatorname{csch}(t)
$$

and thus

$$
P=x e^{-t}-\frac{e^{t}+e^{-t}}{e^{t}-e^{-t}}\left(X-x e^{-t}\right)=x \operatorname{csch}(t)-X \operatorname{coth}(t) .
$$

In Step I of Algorithm 1 we solve the PMP (11) and (12). It is equivalent to finding $X$ that satisfies at least one set of the following conditions

$$
\begin{aligned}
& \left\{\begin{array}{l}
X=\frac{2 x}{e^{t}+e^{-t}}=x \operatorname{sech}(t), \\
X \geq 0 \text { or } X \leq-1,
\end{array}\right. \\
& \text { or }\left\{\begin{array}{l}
X=\frac{2 x+e^{-t}-e^{t}}{e^{t}+e^{-t}}=x \operatorname{sech}(t)-\tanh (t), \\
-1<X<0,
\end{array}\right. \\
& \text { or } X=0, \\
& \text { or } X=-1 .
\end{aligned}
$$

The optimal cost in Step II is

$$
\begin{aligned}
J & =\frac{C^{2}}{2}\left(e^{2 t}-1\right)-\frac{(x-C)^{2}}{2}\left(e^{-2 t}-1\right)+G(X) \\
& =\frac{x^{2}+X^{2}}{2} \operatorname{coth}(t)-x X \operatorname{csch}(t)+G(X)
\end{aligned}
$$

for $t>0$ where $G$ is a continuous function satisfying $G^{\prime}=g$ almost everywhere.

The solution of the conservation law (28) using Algorithm 2 is shown in Figure 7 , The rarefaction and shock waves in the solution are clearly shown in the figure. 


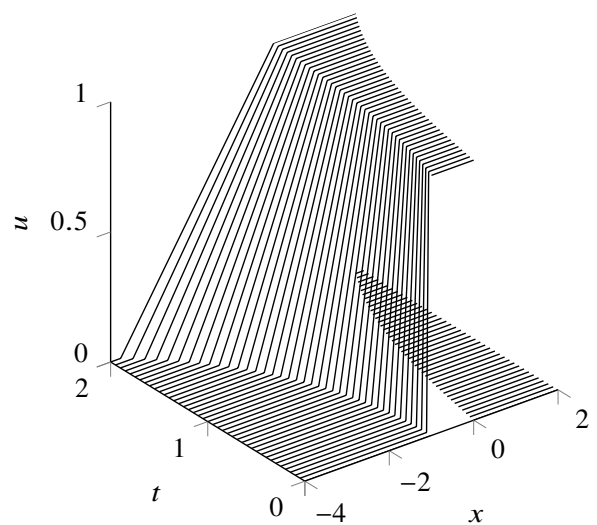

(a)

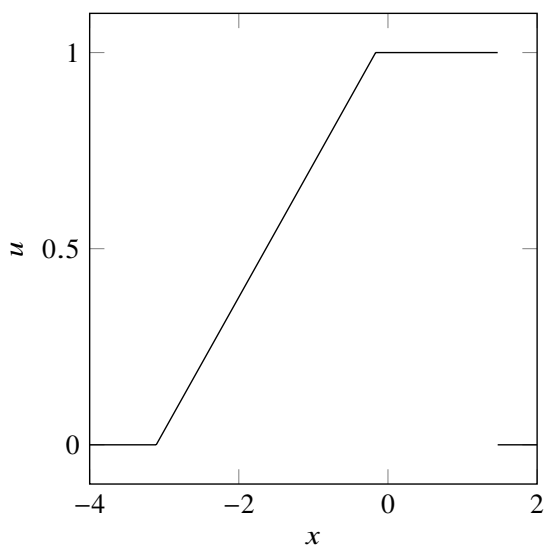

(b)

Fig. 7: The solution of the conservation law with a space-dependent flux given in Example 6 Here (a) is a waterfall plot of the solution and (b) is the solution for $t=1.8$.

Example 7 The follow example is adopted from Zhang and Liu [30]. Consider the conservation law with a space-dependent flux

$$
\begin{aligned}
u_{t}+\left(\left(1+\frac{u}{a}\right) u\right)_{x} & =0 & & \text { in } \mathbb{R} \times(0, T), \\
u & =-\frac{e^{-10 x}}{10} & & \text { on } \mathbb{R} \times\{t=0\}, \\
a & =e^{-10 x} & & \text { on } \mathbb{R} .
\end{aligned}
$$

It can be shown that the problem has no shocks and the unique solution is

$$
u=-\frac{e^{-10 x}}{1+9 e^{-10 t}} .
$$

Again, we use Algorithm 1 to compute the unique minimum value solution. The associated Lagrangian is the Legendre transform of $F \circ(-1)$,

$$
L(x, \alpha)=\frac{a(x)}{4}(\alpha+1)^{2} .
$$

The Hamiltonian is

$$
H(x, p, \alpha)=p \alpha+\frac{a(x)}{4}(\alpha+1)^{2}
$$

and the optimal control satisfies

$$
\alpha^{*}=-1-\frac{2 p}{a(x)} .
$$

Then, it is straightforward to derive the PMP (11)

$$
\begin{aligned}
\dot{\boldsymbol{x}}(r) & =-1-2 e^{10 \boldsymbol{x}(r)} \boldsymbol{p}(r), \\
\dot{\boldsymbol{p}}(r) & =10 e^{10 \boldsymbol{x}(r)}(\boldsymbol{p}(r))^{2}, \\
\boldsymbol{x}(0) & =x, \\
\boldsymbol{p}(t) & =-\frac{e^{-10 \boldsymbol{x}(t)}}{10} .
\end{aligned}
$$


It is a two-point BVP of differential equations. For the purpose of testing a numerical implementation of Algorithm 1, we do not transform (32) into algebraic equations, although the differential equations can be explicitly solved. Instead, we use Chebfun to solve the BVP (32) as described in Birkisson and Driscoll [2]. Due to the uniqueness of solutions, Step II in Algorithm 1 is unnecessary. The Chebfun implementation of damped Newton method is used for the BVP solver with the standard termination criteria and the standard error tolerance of $10^{-10}$ is used. The computation is carried out on a uniform grid of $30 \times 30$ points in the region $[0,1] \times[0.1,0.6]$ with a maximum absolute error of $1.33 \times 10^{-13}$ is observed.

\section{Conclusions}

Using optimal control theory, our study yields an algorithm and implementation for finding the minimum value solution of a scalar convex conservation laws, pointwise. It is proved that in the case of a space-independent flux function the minimum value solution is the entropy solution, thus providing a generalization of the Lax-Oleinik formula. Numerical results show good agreement of solutions from the proposed algorithm with that of analytical solutions and the finite volume method.

\section{A Example using Implementation 1}

As an example of using Implementation 1 we present the code used in Example 1 to generate Figure 1 .

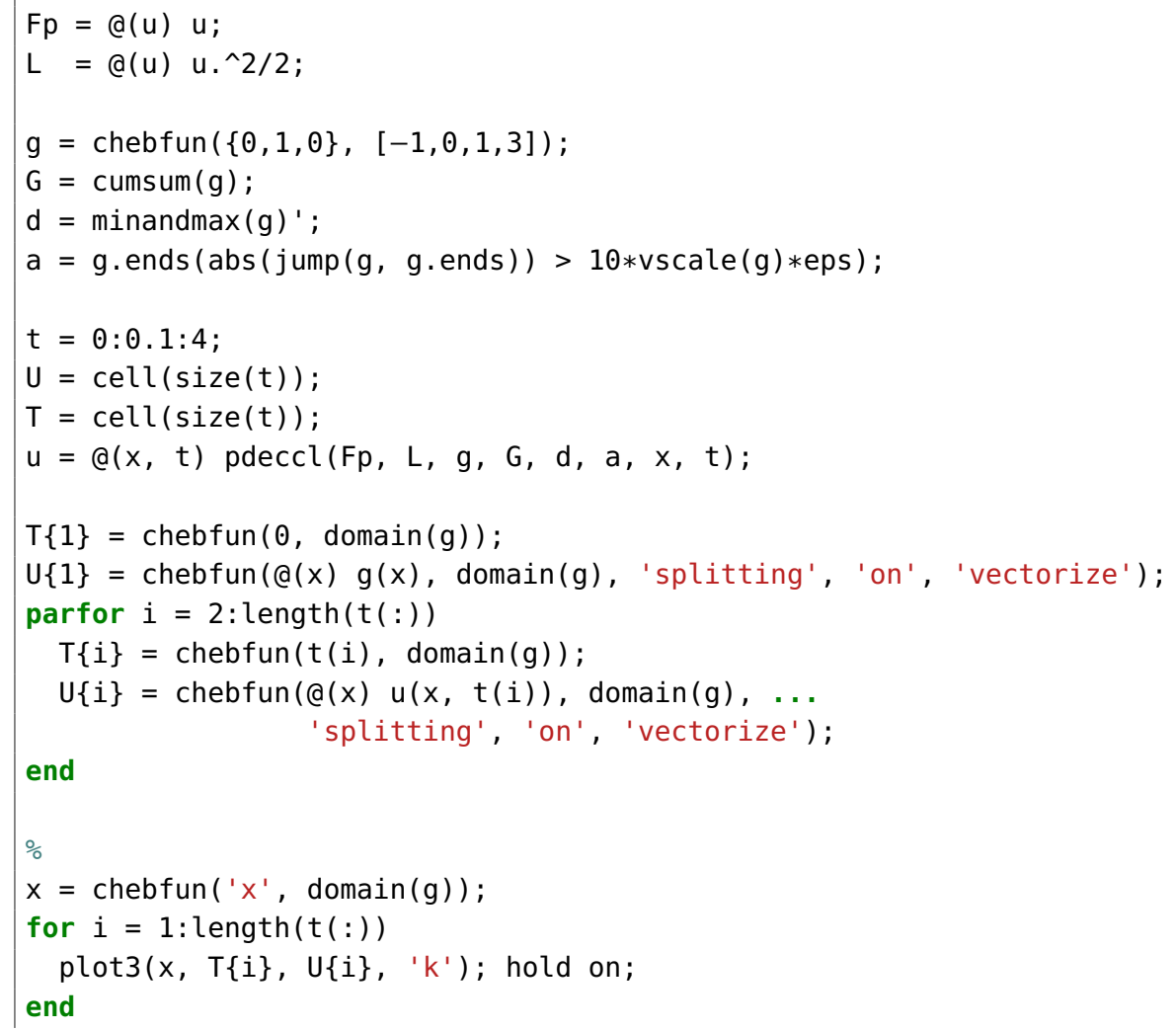




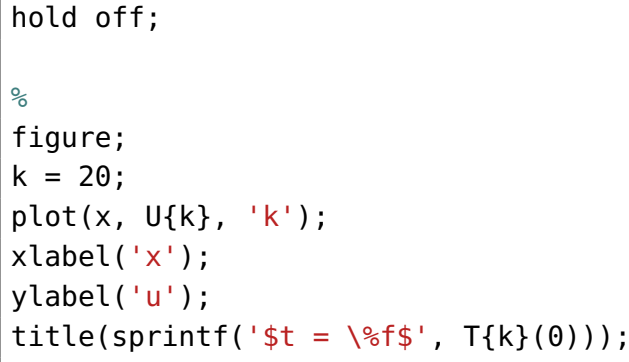

Here we give the point-wise solver pdeccl to chebfun to generate polynomial approximations of the solution, $\mathrm{U}$, for various time instances, $\mathrm{T}$.

Acknowledgements This work was supported in part by AFOSR, NRL, and DARPA. Thanks to Maurizio Falcone for his insights on fast Legendre-Fenchel transform.

\section{References}

1. Chebfun guide. Pafnuty Publications, Oxford (2014). URL http://www. chebfun.org/docs/guide/

2. Birkisson, A., Driscoll, T.A.: Automatic Fréchet differentiation for the numerical solution of boundary-value problems. ACM Transactions on Mathematical Software 38(4), 26:1-26:29 (2012). doi $10.1145 / 2331130.2331134$

3. Boyd, J.P.: Computing zeros on a real interval through Chebyshev expansion and polynomial rootfinding 40(5), 1666-1682 (2002). doi $10.1137 / \mathrm{s} 0036142901398325$

4. Chow, Y.T., Darbon, J., Osher, S., Yin, W.: Algorithm for overcoming the curse of dimensionality for certain non-convex Hamilton-Jacobi equations, projections and differential games. Tech. Rep. UCLA CAM 16-27, University of California, Los Angeles, Department of Mathematics, Group in Computational Applied Mathematics (2016). URL ftp://ftp.math.ucla.edu/pub/camreport/cam16-27.pdf (revised August 2016)

5. Clawpack Development Team: Clawpack software (2016). URL http://www.clawpack.org Version 5.3.1

6. Corrias, L., Falcone, M., Natalini, R.: Numerical schemes for conservation laws via Hamilton-Jacobi equations 64(210), 555 (1995). doi 10.1090/s0025-5718-1995-1265013-5

7. Dafermos, C.M.: Hyperbolic Conservation Laws in Continuum Physics. Grundlehren der mathematischen Wissenschaften, 3 edn. Springer Berlin Heidelberg (2010). doi 10.1007/978-3-642-04048-1

8. Daganzo, C.F.: A variational formulation of kinematic waves: Basic theory and complex boundary conditions. Transportation Research Part B: Methodological 39(2), 187-196 (2005). doi $10.1016 / j . t r b .2004 .04 .003$

9. Darbon, J., Osher, S.: Algorithms for overcoming the curse of dimensionality for certain Hamilton-Jacobi equations arising in control theory and elsewhere. ArXiv e-prints (arXiv:1605.01799) (2016). URL https://arxiv.org/abs/1605.01799

10. Evans, L.: Partial Differential Equations. American Mathematical Society (2010). doi 10.1090/gsm/019

11. Good, I.J.: The colleague matrix, a Chebyshev analogue of the companion matrix 12(1), 61-68 (1961). doi $10.1093 /$ qmath/12.1.61

12. Kang, W., Wilcox, L.: A causality free computational method for HJB equations with application to rigid body satellites. In: AIAA Guidance, Navigation, and Control Conference, AIAA 2015-2009. American Institute of Aeronautics and Astronautics (2015). doi 10.2514/6.2015-2009

13. Kang, W., Wilcox, L.C.: Mitigating the curse of dimensionality: Sparse grid characteristics method for optimal feedback control and HJB equations. ArXiv e-prints (arXiv:1507.04769) (2015). URL https://arxiv.org/abs/1507.04769

14. Lax, P.D.: Hyperbolic Systems of Conservation Laws and the Mathematical Theory of Shock Waves. Society for Industrial \& Applied Mathematics (1973). doi 10.1137/1.9781611970562

15. LeVeque, R.J.: Numerical Methods for Conservation Laws. Springer Science + Business Media (1992). doi 10.1007/978-3-0348-8629-1

16. LeVeque, R.J.: Finite Volume Methods for Hyperbolic Problems. Cambridge University Press (2002). doi $10.1017 /$ cbo9780511791253 
17. Lighthill, M.J., Whitham, G.B.: On kinematic waves. II. A theory of traffic flow on long crowded roads. Proceedings of the Royal Society A: Mathematical, Physical and Engineering Sciences 229(1178), 317-0345 (1955). doi $10.1098 /$ rspa.1955.0089

18. Luke, J.C.: Mathematical models for landform evolution. Journal of Geophysical Research 77(14), 2460-2464 (1972). doi 10.1029/jb077i014p02460

19. Newell, G.F.: A simplified theory of kinematic waves in highway traffic, part I: General theory. Transportation Research Part B: Methodological 27(4), 281-287 (1993). doi 10.1016/0191-2615(93)90038-c

20. Osher, S., Fedkiw, R.: Level Set Methods and Dynamic Implicit Surfaces. Springer-Verlag, New York (2003). doi 10.1007/978-0-387-22746-7

21. Osher, S., Sethian, J.A.: Fronts propagating with curvature-dependent speed: Algorithms based on Hamilton-Jacobi formulations. Journal of Computational Physics 79(1), 12-49 (1988). doi 10.1016/00219991(88)90002-2

22. Osher, S., Shu, C.W.: High-order essentially nonoscillatory schemes for Hamilton-Jacobi equations. SIAM Journal on Numerical Analysis 28(4), 907-922 (1991). doi 10.1137/0728049

23. Pachón, R., Platte, R.B., Trefethen, L.N.: Piecewise-smooth chebfuns 30(4), 898-916 (2009). doi 10.1093/imanum/drp008

24. Qiu, J.M., Shu, C.W.: Convergence of Godunov-type schemes for scalar conservation laws under large time steps. SIAM Journal on Numerical Analysis 46(5), 2211-2237 (2008). doi 10.1137/060657911

25. Richards, P.I.: Shock waves on the highway. Operations Research 4(1), 42-51 (1956). doi 10.1287/opre.4.1.42

26. Specht, W.: Die lage der nullstellen eines polynoms. III 16(5-6), 369-389 (1957). doi 10.1002/mana.19570160509

27. The MathWorks, Inc.: MATLAB R2014b. Natick, Massachusetts, United States (2014)

28. Trefethen, L.N.: Approximation Theory and Approximation Practice. Society for Industrial and Applied Mathematics, Philadelphia, PA, USA (2012). URL http://www . chebfun. org/ATAP/

29. Wright, G.B., Javed, M., Montanelli, H., Trefethen, L.N.: Extension of Chebfun to periodic functions 37(5), C554-C573 (2015). doi 10.1137/141001007

30. Zhang, P., Liu, R.X.: Hyperbolic conservation laws with space-dependent fluxes: II. General study of numerical fluxes 176(1), 105-129 (2005). doi $10.1016 /$ j.cam.2004.07.005 